\title{
A Linear "Head-to-Tail" Chlorido-Bridged Didysprosium Single-Molecule Magnet Exhibiting 100-s Blocking Temperature near $8 \mathrm{~K}$
}

\author{
Xia-Li Ding, Yuan-Qi Zhai, Qian-Cheng Luo, Chao Wu, Yan-Zhen Zheng1*
}

1 Frontier Institute of Science and Technology (FIST), Xi'an Jiaotong University Shenzhen Research School, State Key Laboratory for Mechanical Behavior of Materials, MOE Key Laboratory for Nonequilibrium Synthesis and Modulation of Condensed Matter, Xi'an Key Laboratory of Sustainable Energy and Materials Chemistry, and School of Chemistry, Xi'an Jiaotong University, 99 Yanxiang Road, Xi'an, 710054, Shaanxi.

*Corresponding Author(s): Yan-Zhen Zheng: zheng.yanzhen@xjtu.edu.cn.

\begin{abstract}
Although single-molecule magnets (SMMs) have been considered as the smallest entity to store binary information, the ability to retain the magnetization in one direction for more than 100 seconds is still below liquid nitrogen boiling point ( $77 \mathrm{~K})$. One of the effective ways to enhance the magnetic blocking temperature is to couple the anisotropic magnetic centers in a proper manner. However, this has been only succeeded in the radical-bridged SMMs so far. For non-radical bridges the 100 -second blocking temperature is still not seen. Here we report the best non-radical bridged dimeric SMM - $\left\{\left[\mathrm{Dy}\left(\mathrm{O}^{\mathrm{t}} \mathrm{Bu}\right)(4-\right.\right.$ $\mathrm{NH}_{2}$ py $\left.\left.)_{5}\right]_{2}(\mu-\mathrm{Cl})\right\}\left[\mathrm{BPh}_{4}\right]_{3} \mathbf{1}$ - with an energy barrier to magnetization reversal of $1302(6) \mathrm{K}$ and 100 second blocking temperature near $8 \mathrm{~K}$. Compared to the similar local $C_{5 \mathrm{v}}$ symmetric mononuclear complex $\left[\mathrm{Dy}\left(\mathrm{O}^{\mathrm{t}} \mathrm{Bu}\right) \mathrm{Cl}(\mathrm{py})_{5}\right] \mathrm{BPh}_{4} \mathbf{2}$, the much higher performance of dimeric $\mathbf{1}$ is attributed to the pseudo- $D_{5 h}$ symmetry and "head-to-tail" linked Dy(III) principle magnetic via the collinear Dy-Cl-Dy exchange-coupling pathway.
\end{abstract}

Keywords. Dysprosium, dinuclear, exchange-coupling, chlorido-bridge, single-molecule magnets 


\section{Introduction}

A molecule which can perform the domain function of traditional magnetic materials to store bits of 0 and 1 is perceived as the smallest entity for information storage. Such molecules are called as singlemolecule magnets (SMMs). ${ }^{1-4}$ To implement such function the spins within the molecule should be able to maintain in either up or down direction for a certain time. For traditional superparamagnetic materials this time is defined as 100 seconds and the corresponding temperature is called the blocking temperature (hereafter $T_{\mathrm{B}}{ }^{100 \mathrm{~s}}$ ). ${ }^{5}$ Though the recent dysprosocenium-based SMM shows magnetic hysteresis (hereafter $T_{\mathrm{B}} \mathrm{H}$, note that $T_{\mathrm{B}} \mathrm{H}$ is field sweeping rate dependent) above the boiling point of liquid nitrogen $\left(T_{\mathrm{B}} \mathrm{H}=80 \mathrm{~K}\right)$ the $T_{\mathrm{B}}{ }^{100 \mathrm{~s}}$ is actually at about $65 \mathrm{~K} \cdot{ }^{6}$ Hence, to have more pragmatic applications such as miniaturized spintronic devices and quantum computation, the $T_{\mathrm{B}}{ }^{100 \mathrm{~s}}$ of SMMs still needs to be further enhanced.7-12

As the energy barrier for magnetization reversal $\left(U_{\text {eff }}\right)$ is the premise to obtain high $T_{\mathrm{B}} 100$ s, lanthanide ions with large crystal field splitting are competent.13-15 In this regard, the dysprosium(III) ion, which has large ground magnetic moment of ${ }^{6} \mathrm{H}_{15 / 2}$ ground state is believed to be the best candidate. ${ }^{16-18}$ However, to further enhance the $T_{\mathrm{B}}{ }^{100 \mathrm{~s}}$ still remains challenging. Several strategies were adopted to construct the better-performance SMMs at present. One is to create an axial symmetric environment around Dy(III) ions. ${ }^{19-21}$ Typical examples include the dysprosocenium family ${ }^{22-24}$ and the pentagonalbipyramidal (PB) family. ${ }^{25-27}$ Right now, the former system exhibits great success in the combination of magnetic axiality and stiffness of the molecule thanks to the core pentadentate cyclopentadienyl (Cp) ligands. For the PB Dy(III) SMMs, the strong axial anisotropy is defined by the $C_{5}$ axis ${ }^{28-30}$ as exemplified by the complex $\left[\mathrm{Dy}\left(\mathrm{O}^{\mathrm{t} B u}\right)_{2}(\mathrm{py})_{5}\right] \mathrm{BPh}_{4}$ with $U_{\text {eff }}=1815 \mathrm{~K}$ and $T_{\mathrm{B}}{ }^{100 \mathrm{~s}} \approx 12 \mathrm{~K} .{ }^{26}$ But this family has much lower $T_{\mathrm{B}} 100 \mathrm{~s}$ than $\mathrm{Cp}$ one, which is believed due to the enormously reduced quantum tunneling of the magnetization (QTM) at zero field. ${ }^{31-34}$

Another strategy to enhance $T_{\mathrm{B}}$ is to apply suitable magnetic coupling between the metal centers, 
where generates an exchange bias field to restrain the QTM under zero filed. ${ }^{35-38}$ Introducing magnetic interactions mediated via radical bridges has been the most effective method towards stronger coupling reported until now (Tables 1 and S1). ${ }^{39,40}$ For example, the $\mu-\mathrm{N}_{2}$. bridged $\left\{\mathrm{Tb}_{2}\right\}$ complexes $\left.\left[\left\{\left(\mathrm{Me}_{3} \mathrm{Si}\right)_{2} \mathrm{~N}\right)_{2} \mathrm{~Tb}(\mathrm{THF})\right\}_{2}\left(\mu-\mathrm{N}_{2} \cdot\right)\right]$ with $U_{\text {eff }}=154 \mathrm{~K}$ and $T_{\mathrm{B}} 100 \mathrm{~s}$ of $14 \mathrm{~K}$ and $\left[\left(\mathrm{CpMe}^{4 \mathrm{H}_{2} \mathrm{~Tb}}\right)_{2}\left(\mu-\mathrm{N}_{2} \cdot\right)\right]^{-}$with $U_{\text {eff }}$ $=395 \mathrm{~K}$ and $T_{\mathrm{B}} 100 \mathrm{~s}$ of $20 \mathrm{~K}$; while interestingly, their analogue $\left\{\mathrm{Dy}_{2}\right\}$ complexes show both lower $U_{\text {eff }} \sim$ $170 \mathrm{~K}$ and $T_{\mathrm{B}}{ }^{100 \mathrm{~s}} \sim 8 \mathrm{~K}^{40,41}$ More recently, these numbers have been broken by the single-electron metalmetal bonded endohedral dimetallofullerenes via physically arc-discharge, $\mathrm{Ln}_{2} @ \mathrm{C}_{80}\left(\mathrm{CH}_{2} \mathrm{Ph}\right)$. For $\mathrm{Ln}=\mathrm{Tb}$, $U_{\text {eff }}=799 \mathrm{~K}$ and $T_{\mathrm{B}}{ }^{100 \mathrm{~s}} \approx 25 \mathrm{~K}$; for $\mathrm{Ln}=\mathrm{Dy}, U_{\text {eff }}=613 \mathrm{~K}$ and $T_{\mathrm{B}}{ }^{100 \mathrm{~s}} \approx 18 \mathrm{~K} .42,43$

Although the radical bridge is capable for creating strong exchange-coupling interaction between the 4f metal ions, handling these reactive units is very difficult. Moreover, the radical ligand is not easy to modify if the magnetic exchange coupling needs to regulate. 44,45 We therefore chose to explore the possibility instead of employing more controllable non-free radical bridged ligands to achieve a similar effect. However, for non-radical bridges, dihydroxy-bridge and dinitrogen-bridge are the most common ones in previously reported examples. ${ }^{46,47}$ But with such bridges the dimeric complexes featuring unregular configurations usually suffer the non-collinear alignment of the magnetic principle axes and the exchange-coupling direction. Even worse, the adjacent magnetic ion virtually forms a transverse field that can cause QTM effect to the neighboring metal centers. Hence the highest $T_{\mathrm{B}} 100 \mathrm{~s}$ of non-radical bridged SMMs is not observable. So far, the highest observed $T_{\mathrm{B}} \mathrm{H}$ is $16 \mathrm{~K}$ with a fast sweep rate of 200 Oe/s (See Supporting Information Table S1) ${ }^{38}$

Unlike other cases, the recently reported $3 d-4 f \operatorname{SMM}\left\{\mathrm{Dy}\left[15-\mathrm{MC}_{\mathrm{Cu}}-5\right]\right\}_{2}\left(\mathrm{MC}=\mathrm{Cu}_{5}\right.$ metallacrown $)$ by linking two Dy[15-MC $\left.\mathrm{Mu}_{\mathrm{Cu}}-5\right]$ units with a single hydroxide bridge yields the double-decker complex with a slightly distorted Dy-O-Dy linking angle of $158.2^{\circ}$. Such a "head-to-tail" bridging style successfully enhances the $U_{\text {eff }}$ up to $894 \mathrm{~K}$ and magnetic hysteresis remains opening up to $6 \mathrm{~K}(200 \mathrm{Oe} / \mathrm{s})$ without a sudden drop at zero field,48 indicating the axial ferromagnetic Dy-Dy coupling somewhat restricts the fast QTM effect and holds the largest effective energy barriers in reported d-f SMMs. 
Table. 1 Key magnetic parameters of top dinuclear lanthanide SMMs.

\begin{tabular}{|c|c|c|c|c|c|}
\hline Complex & $U_{\text {eff }} / \mathbf{K}$ & $T_{\mathrm{H}} / \mathrm{K}$ & Sweep rate / Oes ${ }^{-1}$ & $T_{\mathrm{B}}{ }^{100 \mathrm{~s}} / \mathrm{K}$ & Ref \\
\hline \multicolumn{6}{|c|}{ Radical bridged $\mathbf{T b}_{2}$} \\
\hline $\mathrm{Tb}_{2} @ \mathrm{C}_{80}\left(\mathrm{CH}_{2} \mathrm{Ph}\right)$ & 799 & 27 & 95 & 25.2 & 43 \\
\hline$\left[\left(\mathrm{Cp}^{\mathrm{Me} 4 \mathrm{H}}{ }_{2} \mathrm{~Tb}\right)_{2}\left(\mu-\mathrm{N}_{2} \cdot\right)\right]^{-}$ & 395 & 24 & 100 & 20 & 40 \\
\hline$\left\{\left[\left(\mathrm{Me}_{3} \mathrm{Si}\right)_{2} \mathrm{~N}\right]_{2}(\mathrm{THF}) \mathrm{Tb}\right\}_{2}\left(\mu-\mathrm{N}_{2}{ }^{3-}\right)$ & 326 & 14 & 100 & 14 & 41 \\
\hline \multicolumn{6}{|c|}{ Radical bridged $\mathrm{Dy}_{2}$} \\
\hline $\mathrm{Dy}_{2} @ \mathrm{C}_{80}\left(\mathrm{CH}_{2} \mathrm{Ph}\right)$ & 613 & 21 & 95 & 18 & 43 \\
\hline$\left\{\left[\left(\mathrm{Me}_{3} \mathrm{Si}\right)_{2} \mathrm{~N}\right]_{2}(\mathrm{THF}) \mathrm{Dy}\right\}_{2}\left(\mu-\mathrm{N}_{2}{ }^{3-}\right)$ & 178 & 8 & 80 & 8.3 & 41 \\
\hline$\left[\left(\mathrm{Cp}^{\mathrm{Me} \mathrm{H}_{2} \mathrm{Dy}}\right)_{2}\left(\mu-\mathrm{N}_{2} \cdot\right)\right]^{-}$ & 154 & 8 & 100 & 6.6 & 40 \\
\hline$\left[\left(\mathrm{Cp}^{*}{ }_{2} \mathrm{Dy}\right)_{2}(\mu-\mathrm{bpym} \cdot)\right]$ & 126 & 7 & 20 & - & 35 \\
\hline \multicolumn{6}{|c|}{ Non radical bridged $\mathrm{Dy}_{2}$} \\
\hline 1 & 1302 & 20 & 10 & 8 & This work \\
\hline$\left(\mathrm{PPh}_{4}\right)\left[\mathrm{Dy}_{2}(\mathrm{bbpen})_{2}\left\{\mathrm{Co}(\mathrm{CN})_{6}\right\}\right]$ & 975 & 16 & 200 & - & 38 \\
\hline$\left(\mathrm{PPh}_{4}\right)\left[\mathrm{Dy}_{2}(\mathrm{bbpen})_{2}\left\{\mathrm{Fe}(\mathrm{CN})_{6}\right\}\right]$ & 659 & 15 & 200 & - & 38 \\
\hline$\left[\mathrm{Dy}(\mu-\mathrm{OH})(\mathrm{DBP})_{2}(\mathrm{THF})\right]_{2}$ & 721 & 8 & 200 & - & 46 \\
\hline$\left[\mathrm{Dy}\left(\mathrm{Cy}_{2} \mathrm{~N}\right)_{2}(\mu-\mathrm{Cl})(\mathrm{THF})\right]_{2}$ & 623 & 2 & 10 & - & 47 \\
\hline$\left\{\mathrm{Dy}\left[15-\mathrm{MC}_{\mathrm{Cu}}-5\right]\right\}_{2}$ & 894 & 6 & 200 & - & 48 \\
\hline
\end{tabular}

We reason if the exchange-coupling direction can be perfectly aligned in the axial position of the individual metal center the QTM effect should be further reduced. However, this simple idea has not been succeeded because the coordination geometry of the lanthanide is very flexible considering the large radii and ionic bonding nature of the lanthanide ions. ${ }^{48-50}$ Herein, by using the equatorial 4aminopyridine (4- $\mathrm{NH}_{2}$ py) ligand we successfully isolated a dimeric complex $\left\{\left[\mathrm{Dy}\left(\mathrm{O}^{\mathrm{t}} \mathrm{Bu}\right)\left(4-\mathrm{NH}_{2} \mathrm{py}\right)\right]_{2}(\mu-\right.$ $\mathrm{Cl})\}\left[\mathrm{BPh}_{4}\right]_{3}(\mathbf{1})$, which shows a propeller-like $D_{5 \mathrm{~d}}$ symmetry with an unprecedented linear Dy-Cl-Dy linkage. The dimeric compound 1 shows $U_{\text {eff }}$ of $1302(6) \mathrm{K}$ and 100 -s $T_{\mathrm{B}} \approx 8 \mathrm{~K}$ as well as $T_{\mathrm{B}} \mathrm{H}=20 \mathrm{~K}$ (sweep rate $=10 \mathrm{Oe} / \mathrm{s}$ ), which is much enhanced compared to the monomeric $C_{5 \mathrm{v}}$ complex $\left[\mathrm{Dy}\left(\mathrm{O}^{\mathrm{tBu}}\right) \mathrm{Cl}(\mathrm{py})_{5}\right] \mathrm{BPh}_{4}(2)$ with $U_{\text {eff }}=896(7) \mathrm{K}$ and $T_{\mathrm{B}}^{\mathrm{H}}=10 \mathrm{~K}$ (sweep rate $\left.=10 \mathrm{0e} / \mathrm{s}\right)$. Thus, 1 is the best performed non-radical bridged dimeric SMM up to date (Tables 1 and S1). Further theoretical insight shows that this is mainly attributed to the regular PB coordination geometry with perfect collinear alignments of both principle magnetic-axes and the exchange coupling direction of two adjacent Dy ions transferred by this $\mu$-Cl bridge. 


\section{Results and Discussion}

\section{Synthesis}

Based on our previous synthetic method of the complex $\left[\mathrm{Dy}\left(\mathrm{O}^{\mathrm{t}} \mathrm{Bu}\right) \mathrm{Cl}(\mathrm{THF})_{5}\right] \mathrm{BPh}_{4},{ }^{25}$ we select the neutral pyridine and more basic 4-aminopyridine (4- $\mathrm{NH}_{2}$ py) to synthesize the similar $C_{5 \mathrm{v}}$ complexes. Interestingly, for pyridine the complex $\left[\mathrm{Dy}\left(\mathrm{O}^{\mathrm{t} B u}\right) \mathrm{Cl}(\mathrm{py})_{5}\right] \mathrm{BPh}_{4} 2$ was successfully isolated; but for 4$\mathrm{NH}_{2}$ py of the targeted $\left[\mathrm{Dy}\left(\mathrm{O}^{\mathrm{t}} \mathrm{Bu}\right) \mathrm{Cl}\left(4-\mathrm{NH}_{2} \mathrm{py}\right)_{5}\right] \mathrm{BPh}_{4}$ was not successful. Instead the dimeric complex $\mathbf{1}$ was isolated (Scheme 1). Though we were not able to isolate $\left[\mathrm{Dy}\left(\mathrm{O}^{\mathrm{t} B u}\right) \mathrm{Cl}\left(4-\mathrm{NH}_{2} \text { py }\right)_{5}\right] \mathrm{BPh}$, but we postulate that the formation of $\mathbf{1}$ is via such an intermediate. We reason that during the reaction two $C_{5 \mathrm{v}}$ precursors $\left[\mathrm{Dy}\left(\mathrm{O}^{\mathrm{t}} \mathrm{Bu}\right) \mathrm{Cl}\left(4-\mathrm{NH}_{2} \text { py }\right)_{5}\right] \mathrm{BPh}_{4}$ react with each other and abstract one chloride ion driven by the $\mathrm{Cl} \cdots \mathrm{H}-\mathrm{N}$ interactions. The remaining chloride acts as the linear bridge for two Dy(III) ions, forming an unprecedented propeller-like $D_{5 d}$-symmetric complex with linear Dy-Cl-Dy linkage (See Supporting Information in detail).

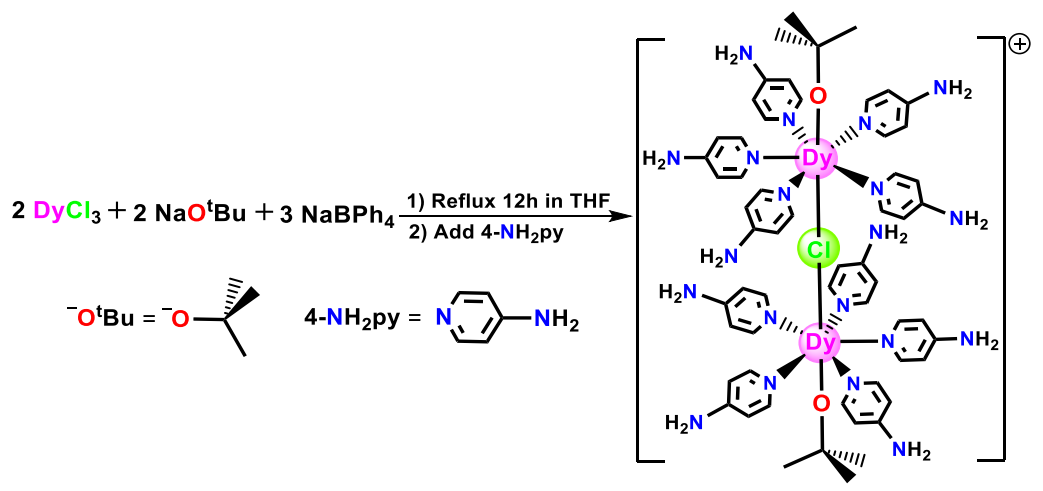

Scheme. 1 The synthetic procedure for complex 1. The balancing [BPh 4 ] anions are omitted in the product.

\section{Structure}

Single crystal X-ray diffraction shows the complex $\mathbf{1}$ comprising of one chloride-bridged dimeric DyIII cation - $\left\{\left[\mathrm{Dy}\left(\mathrm{O}^{\mathrm{t}} \mathrm{Bu}\right)\left(4-\mathrm{NH}_{2} \mathrm{py}\right)_{5}\right]_{2}(\mu-\mathrm{Cl})\right\}^{3+}$ (Fig. 1a) and three charge-balancing $\mathrm{BPh}_{4}^{-}$anions in the crystallographic asymmetric unit (See Supporting Information Table S3). The cationic complex 1 
features a regular pentagonal antiprism with double caps. Hence, the dimeric $\left\{\left[\mathrm{Dy}\left(\mathrm{O}^{\mathrm{t} B u}\right)\left(4-\mathrm{NH}_{2} \mathrm{py}\right)_{5}\right]_{2}(\mu-\right.$ $\mathrm{Cl})\}^{3+}$ cation is propeller-like with formal $D_{5 \mathrm{~d}}$ symmetry. The $\mu$-Cl bridge lies right at the retainversion center between two $C_{5 \mathrm{v}}$-symmetric subunits of the $\left[\mathrm{Dy}\left(\mathrm{O}^{\mathrm{t}} \mathrm{Bu}\right)\left(4-\mathrm{NH}_{2} \mathrm{py}\right)_{5}\right]^{2+}$ complexes (See Supporting Information Table S4), exhibiting perfectly linear linking with Dy-Cl-Dy angle of $179.6^{\circ}$ and Dy $\cdots$ Dy distance of $5.61 \AA$ A. The two alkoxide $\mathrm{O}$ atoms are the most electronegative, leading to very short Dy-O bond lengths of $2.025 \AA ̊$ and $2.052 \AA$ on both sites (Fig. 1b). Moreover, five $\mathrm{N}$ atoms from five 4- $\mathrm{NH}_{2}$ py are coordinated at the equatorial sites with averaged Dy-N distance of $2.54 \AA$ and five equatorial $\mathrm{N}-\mathrm{Dy}-\mathrm{N}$ angles ranging from $69.44(12)^{\circ}$ to $73.41(11)^{\circ}$. Indeed, both $C_{5 \mathrm{v}}$ units are quite regular pentagonal-bipyramidal geometries with an averaged axial $0-\mathrm{Dy}-\mathrm{Cl}$ angle of $177.0^{\circ}$ (See Supporting Information Table S6). Moreover, strong intra- and inter- molecular hydrogen bonds between the

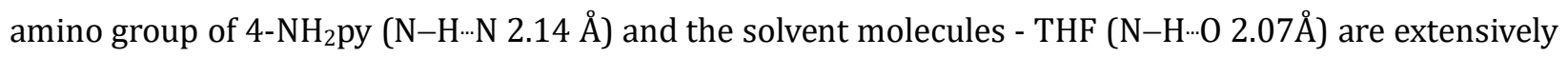
formed (See Supporting Information Figure S3). The shortest intermolecular Dy‥Dy distance is 14.16(2) Å (See Supporting Information Figure S2), which is much longer than intra-molecular Dy...Dy distance of $5.6 \AA ̊$. Thus, the inter-molecular interactions should be negligible. While single crystal X-ray diffraction shows the complex 2 comprising of one mononuclear Dy ${ }^{\mathrm{III}}$ cation - $\left[\mathrm{Dy}\left(\mathrm{O}^{\mathrm{t}} \mathrm{Bu}\right) \mathrm{Cl}(\mathrm{py})_{5}\right]^{+}$and one charge-balancing $\mathrm{BPh}_{4}^{-}$anions in the crystallographic asymmetric unit (See Supporting Information Table S3). The bond lengths and bond angles and geometric coordination configurations of $\mathbf{2}$ are similar to individual dysprosium of complex 1 (See Supporting Information Tables S4 and S6). 

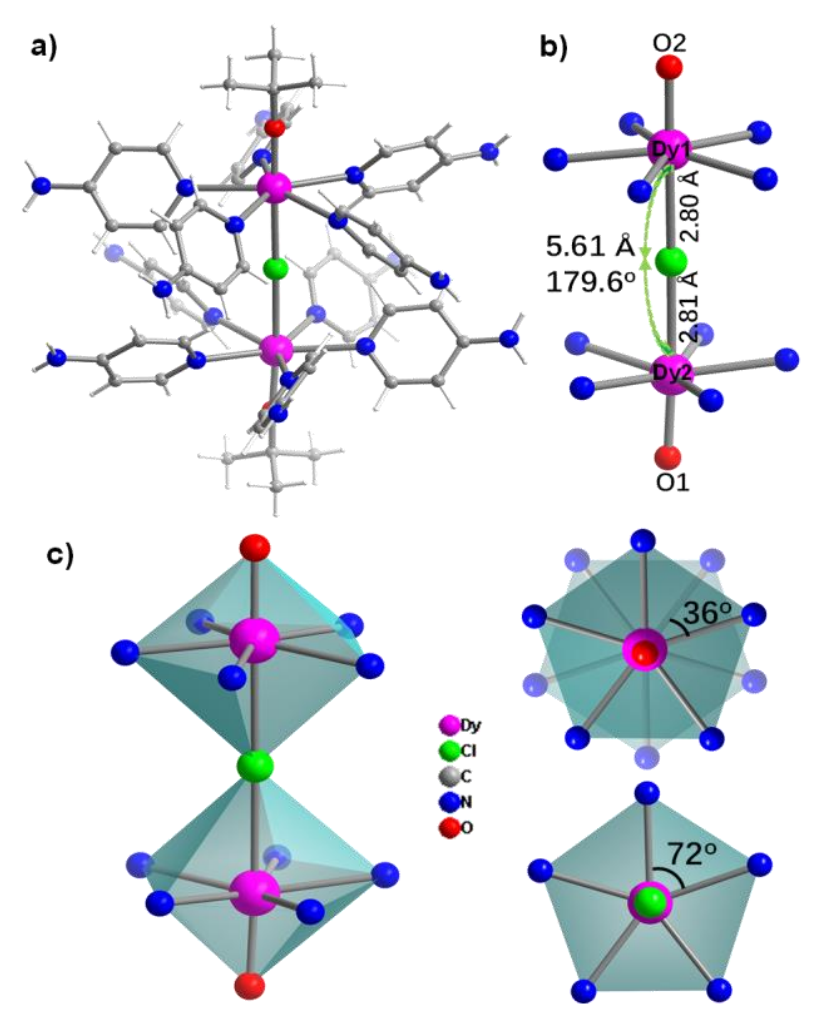

Figure. 1 (a) The crystal structure of $1\left\{\left[\mathrm{Dy}\left(\mathrm{O}{ }^{t} \mathrm{Bu}\right)\left(4-\mathrm{NH}_{2} \mathrm{py}\right)_{5}\right]_{2}(\mu-\mathrm{Cl})\right\}^{3+}$ motif with the $\mathrm{H}$ atoms and anions omitted; (b) The key bond lengths and angles of the complex; (c) Polyhedron of the first coordination shell for Dy III centres seen from front and from top to bottom in lower right.

\section{Magnetic Properties}

Temperature dependent magnetic susceptibilities for 1 (See Supporting Information Figure S4) and 2

(See Supporting Information Figure S11) are measured under $1 \mathrm{kOe}$ dc field in the temperature range of 2-300 K, giving the $\chi T$ value of $27.53 \mathrm{~cm}^{3} \mathrm{~K} \mathrm{~mol}^{-1}$ at $300 \mathrm{~K}$ for 1 . Despite of the splitting of the ${ }^{6} \mathrm{H}_{15 / 2}$ ground state, that value is close to the expected value $\left(28.34 \mathrm{~cm}^{3} \mathrm{~K} \mathrm{~mol}^{-1}\right)$ for the two isolated Dy(III) ions. Upon cooling, $\chi T$ keeps essentially constant with only a little decrease above $20 \mathrm{~K}$. At lower temperature, frozen magnetic behaviors are concluded through the sudden drop of the $\chi T$ product. The lowest $\chi T$ value is $16.75 \mathrm{~cm}^{3} \mathrm{~K} \mathrm{~mol}^{-1}$ at $2 \mathrm{~K}$ (Fig. S4). The descending of the temperature dependent $\chi T$ products may be attributed to three possible reasons: antiferromagnetic coupling, thermal depopulation of low lying crystal field states and magnetic anisotropy. 49,50 
The field $(H)$ dependence of the magnetization $(M)$ increases steadily up to the maximum value of 9.63 $\mu_{\mathrm{B}}$ at $2 \mathrm{~K}$ and $2.5 \mathrm{~T}$ (See Supporting Information Figure S4, insert); additional $M(H)$ measurements with temperatures higher than $T_{\mathrm{H}}$ (up to $150 \mathrm{~K}$ ) show simple paramagnetism for 1 (See Supporting Information Figure S9). All these values are much lower than the theoretical magnetization saturation value of $10 \mu_{\mathrm{B}}$, suggesting the presence of significant magnetic anisotropy. Opened magnetic hysteresis loop was observed up to $20 \mathrm{~K}$ under a scanning rate of $10 \mathrm{Oe} / \mathrm{s}$ (Fig. 2a). The loop was kept open near to zero field with a coercive field $(\mathrm{Hc})$ of about $0.8 \mathrm{~T}$ at $2 \mathrm{~K}$ and appear sheer steps near to zero field, indicating the presence of QTM progress to some extent. And the zero-field-cooled and field-cooled (ZFCFC) magnetizations showed a peak at 11K (Fig. 3, insert). While complex 2 exhibits butterfly-shaped hysteresis loops at a sweeping rate of $10 \mathrm{Oe} / \mathrm{s}$ (Fig. 2b) up to $10 \mathrm{~K}$, the hysteresis loops show no remanence at zero-field. In addition, the zero-field-cooled (ZFC) magnetization shows a peak at 5K, indicating a much lower $T_{\mathrm{B}}^{\mathrm{ZFC}}$ compared to that of $\mathbf{1}$ (See Supporting Information Figure S13, insert).
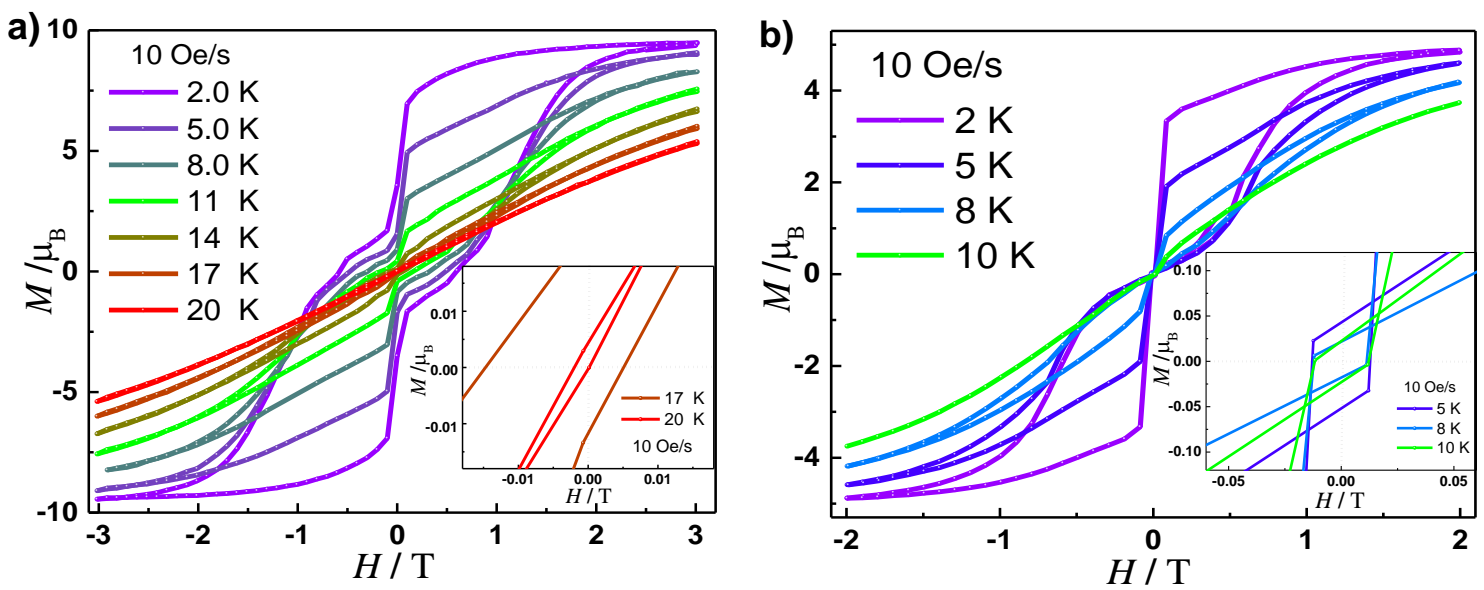

Figure. 2 Magnetic hysteresis loops for 1(a) and 2(b) under a scanning rate of 10 Oe/s.

Significant temperature and frequency dependence in ac susceptibilities under zero dc field were clearly observed for the complex $\mathbf{1}$ and $\mathbf{2}$ at relatively high temperature range. The maxima of both the in-phase $\left(\chi^{\prime}\right)$ and out-of-phase $\left(\chi^{\prime \prime}\right)$ components showed a clear $T$ dependence, revealing the typical slow magnetic relaxation behavior of SMMs for 1 (See Supporting Information Figure S6) and 2 (See Supporting Information Figure S12a). To get the energy barrier for the complex 1 and 2, frequency- 
dependent ac susceptibilities were measured and resonance peaks of $\chi$ " could be observed between $40 \mathrm{~K}$ and $86 \mathrm{~K}$ in 1-1218 $\mathrm{Hz}$ (See Supporting Information Figure S7) for 1, between $22 \mathrm{~K}$ and $52 \mathrm{~K}$ in 1-1218 Hz for 2 (See Supporting Information Figure S12a). The Cole-Cole plots are well fitted with a modified Debye function between $40 \mathrm{~K}$ and $86 \mathrm{~K}$ for 1 (See Supporting Information Figure S10) and for 2 between $22 \mathrm{~K}$ and $52 \mathrm{~K}$ (See Supporting Information Figure S12b) with all the $\alpha$ values less than 0.1 (See Supporting Information Tables S7 and S8), indicating a narrow distribution of relaxation time. As Figure 3 shows, the plots of $\boldsymbol{\tau}^{\mathbf{- 1}}$ vs. $T$ are obtained for the relaxation process by fitting the data with the equation $\boldsymbol{\tau}^{-1}=\boldsymbol{\tau}_{\mathbf{0}}{ }^{-1} \boldsymbol{e}^{-\boldsymbol{U}_{e f f} / \boldsymbol{T}}+\boldsymbol{C} \boldsymbol{T}^{n}$, giving nearly-identical $U_{\text {eff }}$ and $n$ parameters for $\mathbf{1}\left(U_{\mathrm{eff}}=\right.$ 1302(6) $\mathrm{K}, \tau_{0}=7.0 \times 10^{-11} \mathrm{~s}, C=3.12 \times 10^{-5} \mathrm{~s}^{-1} \mathrm{~K}^{-\mathrm{n}}, n=2.8$ ). In the same way, nearly-identical $U_{\mathrm{eff}}$ and $n$ parameters $\left(U_{\text {eff }}=896(7) \mathrm{K}, \tau_{0}=5.0 \times 10^{-12} \mathrm{~s}, C=4.21 \times 10^{-5} \mathrm{~s}^{-1} \mathrm{~K}^{-n}, n=2.93\right.$ ) were got for 2 (See Supporting Information Figure S13). Furthermore, from this fitting result the $T_{\mathrm{B}} 100 \mathrm{~s}$ is calculated to be about $8 \mathrm{~K}$ for $\mathbf{1}$. To double-check this result a dc relaxation method to determine the relaxation time at 8 $\mathrm{K}$ is performed, which leads to $\tau_{8 \mathrm{~K}} \approx 93.5$ seconds (See Supporting Information Figure S10), very close to the fitting result.

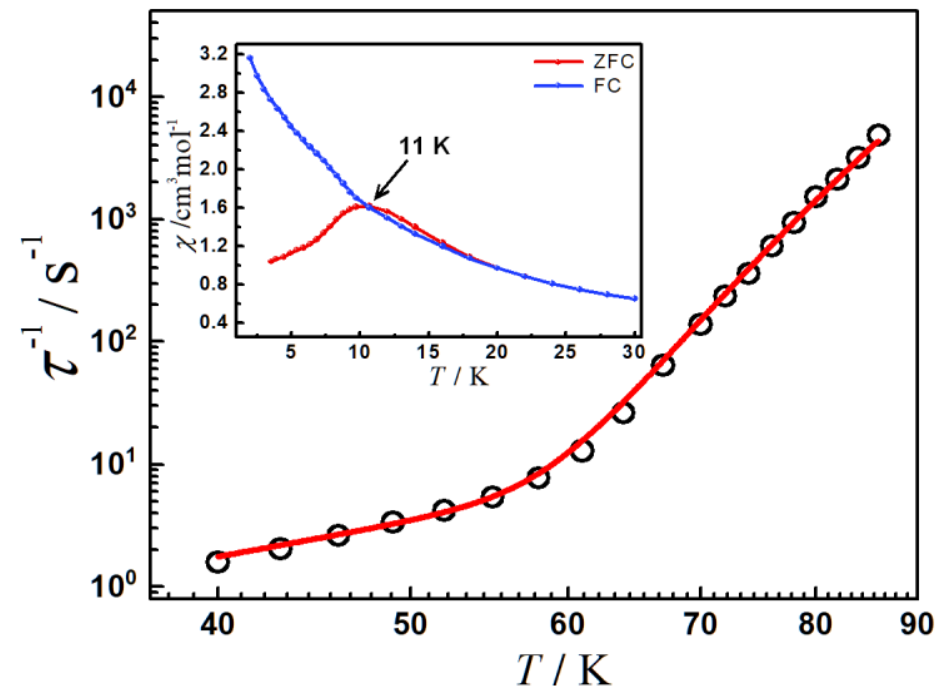

Figure. 3 Plot of temperature-dependent magnetic relaxation times for 1. Insert: Variable temperature magnetic susceptibility of 1 under field-cooled (FC) and zero-field-cooled (ZFC) conditions under a dc field of 1000 Oe. 


\section{Electronic Structure Calculations}

Two individual DyIII fragments (named by Dy1 and Dy2) in 1 were calculated using a widely used methodology, that is complete-active-space self-consistent field (CASSCF) method, based on the OPENMOLCAS software (see Supporting Information for more details). Due to the molecular symmetry, two DyIII ions possess almost the same coordination sphere, featuring similar magnetic structures. The results corroborated axial-type magnetic anisotropy for both complexes with ground state purely ( $\sim 99.4 \%)$ composed of $\mid \pm 15 / 2>$ Kramers Doublets (KDs). The principle magnetic axis for the ground state is almost overlapped with the $\mathrm{O}-\mathrm{Dy}-\mathrm{Cl}$ direction (Fig. 4, insert). Due to the huge negative electrostatic potential provided by the ${ }^{-} \mathrm{O}^{\mathrm{t}} \mathrm{Bu}$ anions, the first and second excited KDs kept the direction similar with ground one and possess ( 98.6\%) $\mid \pm 13 / 2>\left(422 \mathrm{~cm}^{-1}\right.$ in energy) and ( 93.3\%) $\mid \pm 11 / 2>\left(726 \mathrm{~cm}^{-1}\right.$ in energy) wavefunctions, respectively (See Supporting Information Table S9). The other higher-lying KDs were in a strong mixture of states, thus the predicted most efficient magnetic relaxation pathway was via the highly bunched set of states around $880 \sim 946 \mathrm{~cm}^{-1}(1265 \sim 1360 \mathrm{~K})$, which was consistent with experimentally determined $U_{\text {eff. }}$ While for $\mathbf{2}$ the ground and the first excited doublets possess pure wave functions: $99.4 \% \mid \pm 15 / 2>$ and $98.4 \% \mid \pm 13 / 2>$. However, higher doublets from the third one are mixed and $\mathrm{g}_{\mathrm{z}}$ angle becomes $87.01^{\circ}$ in the second excited doublet. Therefore, the relaxation process might go through this state, making the theoretical energy barrier is about 536 568 $\mathrm{cm}^{-1}$ (771 817 K) (See Supporting Information Table S12 and Figure S15), which was in agreement with the experimentally determined $U_{\text {eff. }}$ 


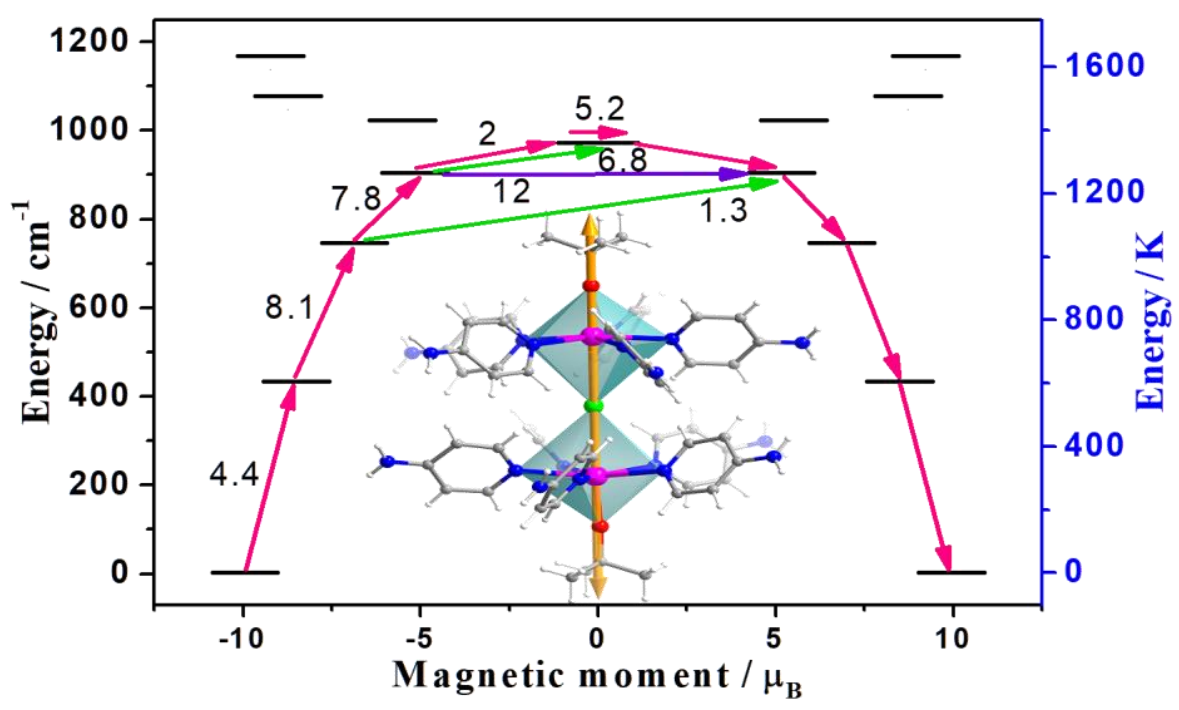

Figure. 4 Ab initio calculated electronic states of the $J=15 / 2$ manifold of the ${ }^{6} H_{15 / 2}$ term of Dy(III) in the complex 1. The principal magnetic axis of the ground Kramer's doublet of Dy1 and Dy2 in the dimer are elucidated by the orange arrow (insert). The connected energy states with the number representing matrix element of the transverse moment are shown by the arrows. The KDs as function of magnetic moments are illustrated by the black line. The purple solid arrow indicates the QTM via ground state and TA-QTM (thermally assisted QTM) via excited states. The green solid arrow presents possible Orbach relaxation mechanism. The mechanism of magnetic relaxation is indicated by the red arrow.

The fragment calculations predicted the Ising limit type for ground KDs of both Dy(III) sites, thus the intra-molecular interactions can be calculated by the following Hamiltonian Equations 1-3:

$$
\begin{gathered}
\widehat{\boldsymbol{H}}_{t o t}=\widehat{\boldsymbol{H}}_{e x}+\widehat{\boldsymbol{H}}_{\text {dip }} \text { (1) } \\
\widehat{\boldsymbol{H}}_{e x}=-2 \boldsymbol{J}_{e x} \tilde{S}_{j} \tilde{\boldsymbol{S}}_{j+1}(2) \\
\widehat{\boldsymbol{H}}_{\text {dip }}=\frac{\boldsymbol{\mu}_{0}}{4 \pi} \sum_{p, q}\left(\frac{\boldsymbol{M}_{p} \cdot \boldsymbol{M}_{q}}{\left|\boldsymbol{R}_{p q}\right|^{3}}-3 \frac{\left(M_{p} \cdot \boldsymbol{R}_{p q}\right)\left(M_{q} \cdot \boldsymbol{R}_{p q}\right)}{\left|\boldsymbol{R}_{p q}\right|^{5}}\right)
\end{gathered}
$$

The simulation of dc magnetic data (See Supporting Information Figure S4) was performed using program POLY_ANISO, and best-fit gave both exchange $\left(U_{\text {ex }}\right)$ and dipolar $\left(U_{\text {dip }}\right)$ interactions in the DyIII-DyIII dimer. The $J_{\text {dip }}$ was directly determined by the vector of two DyIII sites' principle axis (Fig. 4, insert), while $J_{\text {ex }}$ was a complex effect induced by bridge ligands. Within the Lines model, the best-fit gave $J_{\text {dip }}=$ 
$0.02 \mathrm{~cm}^{-1}$ and $J_{\mathrm{ex}}=-0.06 \mathrm{~cm}^{-1}$, thus the total intra-molecular coupling $J_{\mathrm{tot}}=J_{\mathrm{dip}}+J_{\mathrm{ex}}=-0.04 \mathrm{~cm}^{-1}$. According to the Figure S4, the fittings of magnetic susceptibility and magnetization were quite good, which confirmed the small value of $J_{\text {tot. }}$ In this case, we can plot the Zeeman diagram of exchange coupling based spectrum (See Supporting Information Figure S14), with specific interpretation shown in Fig. 5a. The $d_{M} / d_{H}$ vs. $H$ plot in Fig $\mathbf{5 b}$ was obtained from the first derivative of $M$ vs. $H$ plots, which indicates the slop of magnetizations upon raising the magnetic field. Thus, the crossing point between ground and excited states of the Zeeman diagram was calculated at $0.13 \mathrm{~T}$ in Fig 5a, which is close to the turning point of $0.18 \mathrm{~T}$ yielded from the $d_{M} / d_{H}$ vs. $H$ plot in Fig 5b. Above two methods were applied to obtain the magnetic coupling interaction constant. One method is based on the Zeeman effect. Considering that the steps on hysteresis curves are the consequence of level-crossing between the ground state and the first-excited state of the dimer, eqn (2) could be applied to calculate the J value. ${ }^{46,48}$ The first derivative of the magnetization of 1 reveals that the crossing field could be estimated as being $1.8 \mathrm{kOe}$. The other method involves fitting the dc magnetic susceptibility of compound $\mathbf{1}$ during $2 \mathrm{~K}$ to $300 \mathrm{~K}$ using the program POLY_ANISO. Both results indicate that the exchange coupling transferred through the one chloride bridges is weak among the bridged Dy(III) complex. 

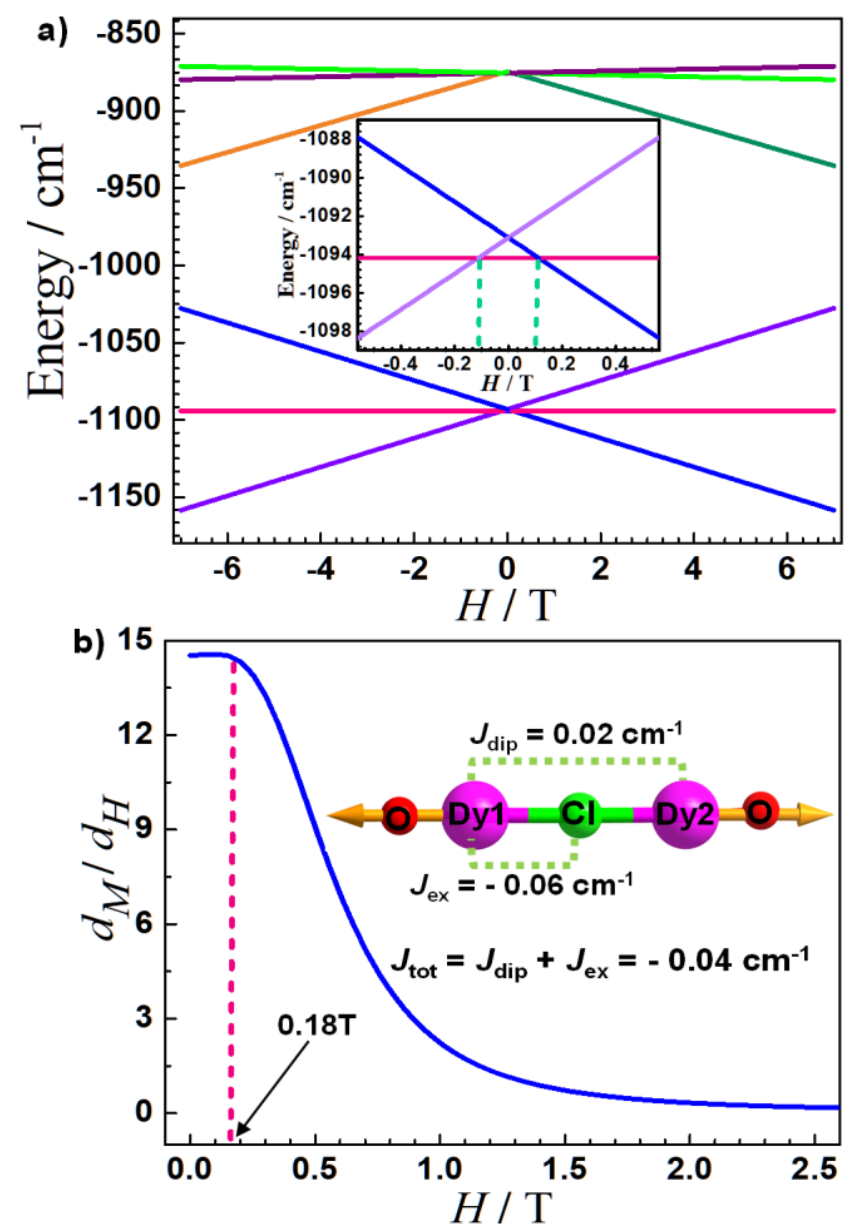

Figure. 5 (a) A stylized depiction of a Zeeman energy diagram for the lowest doublet of an atom (Dy3+) in 1 with a ground state $\left(m_{J}= \pm 15 / 2\right)$ in the presence of an external magnetic field along the z-axis and an arbitrary hyperfine coupling term. Zoomed area shows an avoided crossing between two hyperfine levels that can allow adiabatic passage from $+m_{J}$ to $-m_{J}$ when the external field is swept through the avoided crossing; (b) The first derivtive of the magnetization plot.

It is interesting to compare the magnetic properties of radical and non-radical bridged $\mathrm{Ln}_{2} \mathrm{SMMs}_{\text {. For }}$ radical bridge, such as in the complexes $\mathrm{Ln}_{2} @ \mathrm{C}_{80}\left(\mathrm{CH}_{2} \mathrm{Ph}\right)^{43}$ and $\left[\left(\mathrm{Cp}^{\mathrm{Me}} 4 \mathrm{H}_{2} \mathrm{Ln}\right)_{2}\left(\mu-\mathrm{N}_{2} \cdot\right)\right]^{-40}$ the radical exchange-couplings are so strong that the $U_{\text {eff }}$ is mainly determined by the magnitude of $J_{\text {ex. }}$ More impressively, the magnetic anisotropic axes of the metal ions are collinearly aligned. To this end, the radical between two lanthanides is like super-glue to stick the anisotropic axes in a head-to-tail fashion. But for the non-radical bridged dimeric complexes the bridge is usually neither a single atom nor a single electron except for the recently achieved Dy-CN-M-CN-Dy linkage in the complexes 
$\left(\mathrm{PPh}_{4}\right)\left[\mathrm{Dy}{ }_{2}(\text { bbpen })_{2}\left\{\mathrm{M}(\mathrm{CN})_{6}\right\}\right](\mathrm{M}=\mathrm{Co} / \mathrm{Fe}) \cdot{ }^{38}$ However, though the cyanide can transmit very strong magnetic exchange-couplings such bridge still cannot change the side-by-side style of magnetic principle axes of the Dy(III) ions. As such, the non-radical bridged dimeric SMMs are still single-ion magnetism dominated. In this case, the adjacent magnetic moments in the side-by-side style of $\mathrm{Dy}_{2} \mathrm{dimer}_{\text {can }}$ provide a virtual transverse field to induce the fast QTM at zero field. In this respect, the head-to-tail style of the perfectly linear dimeric Dyz in $\mathbf{1}$ is very scarce, which unambiguously contribute to the high performance of the magnetic blocking and partly restricted QTM effect at zero field. During the review of this paper we realized a dimeric $\left\{\mathrm{Dy}\left[15-\mathrm{MC}_{\mathrm{Cu}}-5\right]\right\}_{2}\left(\mathrm{MC}=\mathrm{Cu}_{5}\right.$ metallacrown) SMM was reported, which shows two Dy[15- $\left.\mathrm{MC}_{\mathrm{Cu}}-5\right]$ units linked by a single hydroxide bridge and yields a distorted "head-to-tail" bridging mode, but the angle of Dy-OH-Dy $\left(158.2^{\circ}\right)$ is not straight enough. Such a bending bridging mode results in a much enhanced energy barrier of $894 \mathrm{~K}$ and opening magnetic hysteresis up to $6 \mathrm{~K}$ $(200 \mathrm{Oe} / \mathrm{s}){ }^{48}$ All these recent progresses reinforce the importance of linear "head-to-tail" bridging mode in designing high-performance SMMs. Just as Tong and co-worker mentioned that combining Ising-type magnetic anisotropy with collinear magnetic interactions in SMMs is a significant synthetic challenge. ${ }^{48}$ Moreover, only those with unpaired electron strongly delocalized on the ligand can enforce the magnetic anisotropy axes of the metal center collinear. For weaker radicals or non-radical bridges the axiality is always weakened. ${ }^{42,43}$ In this regard, the control of the collinearity throughout the complex formed with two local $C_{5 \mathrm{v}}$ symmetry which is bridged by $\mu$-Cl between the magnetic anisotropic axes and the exchange-coupling interactions help improve the performance of SMM on the basis of our previous single $C_{5 \mathrm{v}}$ symmetric complexes. Thus, we estimated that the local $C_{5 \mathrm{v}}$ symmetry and "head-to-tail" arrangement of the Dy(III) principle magnetic axes lead to such performance of $\mathbf{1}$.

\section{Conclusion}

To summarize, we have successfully isolated a propeller-like $D_{5 \mathrm{~d}}$ symmetric Dy(III) dimer with strong magnetic anisotropy by introducing one central $\mu$-chlorido bridge between two $C_{5 \mathrm{v}}$ symmetric units, 
which exhibits a much enhanced effective energy barrier of 1302(6) K and 100-s blocking temperature near $8 \mathrm{~K}$ as well as $T_{\mathrm{B}} \mathrm{H}=20 \mathrm{~K}$. This work achieves the first dimeric Dy-SMM with single anionic bridge and the linear bridging mode in the axial position makes $\mathbf{1}$ the best performed non-radical bridging dimeric SMM. Future control of the magnetic axiality for polynuclear complexes on the basis of maintaining high symmetry should not just align the magnetic principle axes of the metal centers parallel but also the collinearity of the exchange-coupling direction and the principle axes.

\section{Supporting Information}

Supporting Information is available and includes the followings:

- Additional experimental details on the synthesis of compound $\mathbf{1}$ and $\mathbf{2}$

- Cif files 1 and $\mathbf{2}$

- Figures S1-S15

- Tables S1-S13

\section{Conflict of Interest}

There is no conflict of interest to report.

\section{Funding Information}

This work was supported by National Natural Science Foundation of China (nos. 21773130, 21801202 and 21971203), Shenzhen Science and Technology Program (JCYJ20180306170859634), Key Scientific and Technological Innovation Team of Shaanxi Province (2020TD-001), State Key Laboratory for Mechanical Behavior of Materials (20182006), Key Laboratory Construction Program of Xi'an Municipal Bureau of Science and Technology (201805056ZD7CG40), Shaanxi National Science Foundation (No. 2019JQ-016), China Postdoctoral Science Foundation (Nos. 2019T120891 and 2018M643615) and the Fundamental Research Funds for Central Universities. 


\section{Preprint Acknowledgement}

Research presented in this article was posted on a preprint server prior to publication in CCS Chemistry. The corresponding preprint article can be found here: (DOI; Direct Link)

\section{Acknowledgements}

We thank the Instrument Analysis Center of Xi'an Jiaotong University for the assistance from Dr. Gang Chang.

\section{References}

1. Sessoli, R.; Gatteschi, D.; Caneschi, A.; Novak, M.A. Magnetic Bistability in a Metal-ion Cluster. Nature. 1993, 365, 141-143.

2. Leuenberger, M. N. \& Loss, D. Quantum computing in molecular magnets. Nature, 2001, 410, 789-793.

3. Zhu, Zhenhua; Guo, Mei; Li, Xiaolei; Tang, Jinkui. Molecular Magnetism of Lanthanide: Advances and Perspectives. Coord. Chem. Rev. 2019, 378, 350-364.

4. Goodwin, C.A.P; Blocking Like it's Hot: A Synthetic Chemists' Path to High-temperature Lanthanide Single-Molecule Magnets. Dalton Trans. 2020, 49, 14320-14337.

5. Sessoli, R.; Gatteschi, D.; Villain, J. Molecular Nanomagnets; Oxford University Press: Oxford, 2006.

6. Guo, Fusheng; Day, B.M.; Chen, Yancong; Tong, Mingliang; Layfield, R.A. Magnetic Hysteresis up to 80 Kelvin in a Dysprosium Metallocene Single-Molecule Magnet. Science. 2018, 362, 1400-1403.

7. Goodwin, C.A.P.; Ortu, F.; Reta, D.; Chilton, N.F.; Mills, D.P. Molecular Magnetic Hysteresis at 60 Kelvin in Dysprosocenium. Nature. 2017, 548, 439-442. 
8. Gao, Chen; Genoni, A.; Gao, Song; Jiang, Shangda; Soncini, A.; Overgaard, J. Observation of the Asphericity of $4 f$-electron Density and Its Relation to the Magnetic Anisotropy Axis in Single-molecule Magnets. Nat. Chem. 2020, 12, 213-219.

9. Wang, Hailong; Wang, Binwu; Bian, Yongzhong; Gao, Song; Jiang, Jianzhuang. Single-molecule Magnetism of Tetrapyrrole Lanthanide Compounds with Sandwich Multiple-decker Structures. Coord. Chem. Rev. 2016, 306, 195-216.

10. Ma, Yujuan; Hu, Jixiang; Han, Songde; Pan, Jie; Li, Jinhua; Wang, Guoming. Manipulating On/Off Single-Molecule Magnet Behavior in a Dy(III)-Based Photochromic Complex. J. Am. Chem. Soc. 2020, 142, 2682-2689.

11. Gupta, S.K.; Rajeshkumar, T.; Rajaraman, G.; Murugavel, R. An air-stable Dy(III) Single-ion Magnet with High Anisotropy Barrier and Blocking Temperature. Chem. Sci. 2016, 7, 5181-5191.

12. Briganti, M.; Garcia, G.F.; Jung, J.L.; Sessoli, R.; Guennic, B.L.; Totti, F. Covalency and Magnetic Anisotropy in Lanthanide Single Molecule Magnets: the Dy DOTA Archetype. Chem. Sci. 2019, 10, 72337245.

13. Wu, Siguo; Ruan, Zeyu; Huang, Guozhang; Zheng, Jieyu; Vieru, V.; Taran, G.; Wang, Jin; Chen, Yancong; Liu, Junliang; Chibotaru, L.F.; Wernsdorfer, W.; Chen, Xiaoming; Tong, Mingliang. Field-induced Oscillation of Magnetization Blocking Barrier in a Holmium Metallacrown Single-Molecule Magnet. Chem. 2021, 7, $1-11$.

14. Pedersen, K.S.; Ariciu, A.M.; McAdams, S.; Weihe, H.; Bendix, J.; Tuna, F.; Piligkos, S. Toward Molecular 4f Single-ion Magnet Qubits. J. Am. Chem. Soc. 2016, 138, 5801-5804.

15. Gregson, M.; Chilton, N.F.; Ariciu, A.M.; Tuna, F.; Crowe, I.F.; Lewis, W.; Blake, A.J.; Collison, D.; McInnes, E.J.L.; Winpenny, R.E.P.; Liddle, S.T. A Monometallic Lanthanide Bis(methanediide) Single Molecule 
Magnet with a Large Energy Barrier and Complex Spin Relaxation Behaviour. Chem. Sci. 2016, 7, 155165.

16. Parmar, V.S.; Ortu, F.; Ma, Xiaozhou; Chilton, N.F.; Clérac, R.; Mills, D.P.; Winpenny, R.E.P. Probing Relaxation Dynamics in Five-coordinate Dysprosium Single-Molecule Magnets. Chem. Eur. J. 2020, 26, 7774-7778.

17. Ding, Yousong; Han, Tian; Zhai, Yuanqi; Chilton, N.F.; Winpenny, R. E. P.; Zheng, Yanzhen; A Study of Magnetic Relaxation in Dysprosium(III) Single-molecule Magnets. Chem. Eur. J. 2020, 26, 5893-5902.

18. Rinehart, J.D.; Long, J.R. Exploiting Single-ion Anisotropy in the Design of f-element Single-molecule Magnets. Chem. Sci. 2011, 2, 2078-2085.

19. Ding, Xiali; Zhai, Yuanqi; Han, Tian; Chen, Weipeng; Ding, Yousong; Zheng, Yanzhen. A Local $D_{4 \mathrm{~h}}$ Symmetric Dysprosium(III) Single-molecule Magnet with an Energy Barrier Exceeding 2000 K. Chem. Eur. J. 2021, 27, 2623-2627.

20. Liu, Junliang; Chen, Yancong; Zheng, Yanzhen; Lin, Weiquan; Ungur, L.; Wernsdorfer, W.; Chibotaru, L.F.; Tong, Mingliang. Switching the Anisotropy Barrier of a Single-ion Magnet by Symmetry Change From Quasi- $D_{5 \mathrm{~h}}$ to Quasi- $O_{\mathrm{h}}$. Chem. Sci. 2013, 4, 3310-3315.

21. Gupta S.K; Murugavel R. Enriching lanthanide Single-ion Magnetism Through Symmetry and Axiality. Chem. Commun. 2018, 54, 3685-3696.

22. McClain, K.R.; Gould, C.A.; Chakarawet, K.; Teat, S.J.; Groshens, T.J.; Long, J.R.; Harvey, B.G. Hightemperature Magnetic Blocking and Magneto-structural Correlations in a Series of Dysprosium(III) Metallocenium Single-Molecule Magnets. Chem. Sci. 2018, 8492-8503. 
23. Moutet, J.; Schleinitz, J.; Droitte, L.L.; Tricoire, M.; Pointillart, F.; Gendron, F.; Simler, T.; Clavaguéra, C.; Guennic, B.L.; Cador, O.; Nocton, G. Bis-cyclooctatetraenyl Thulium(II): Highly Reducing Lanthanide Sandwich Single Molecule Magnets. Angew. Chem. Int. Ed. 2021, 133, 2-28.

24. Gould, C.A.; McClain, K.R.; Yu, J.M.; Groshens, T.J.; Furche, F.; Harvey, B.G.; Long, J.R. Synthesis and Magnetism of Neutral, Linear Metallocene Complexes of Terbium(II) and Dysprosium(II). J. Am. Chem. Soc. 2019, 141, 12967-12973.

25. Ding, Yousong; Yu, Kexin; Reta, D.; Ortu, F.; Winpenny, R.E.P.; Zheng, Yanzhen; Chilton, N.F. Field- and Temperature-dependent Quantum Tunnelling of the Magnetisation in a Large Barrier Single-molecule Magnet. Nat. Commun. 2018, 9, 3134-3137.

26. Ding, Yousong; Chilton, N.F.; Winpenny, R.E.P.; Zheng, Yanzhen; On Approaching the Limit of Molecular Magnetic Anisotropy: A Near-perfect Pentagonal Bipyramidal Dysprosium(III) Singlemolecule Magnet. Angew. Chem. Int. Edit. 2016, 55, 16071-16074.

27. Liu, Jiang; Chen, Yancong; Liu, Junliang. Vieru, V.; Ungur, L.; Chibotaru, L.F.; Gao, Song; Chen, Xiaoming; Tong, Mingliang. A Stable Pentagonal Bipyramidal Dy(III) Single-ion Magnet with a Record Magnetization Reversal Barrier over 1000 K. J. Am. Chem. Soc. 2016, 138, 5441-5450.

28. McClain, K.R.; Gould, C.A.; Chakarawet, K.; Teat, S.J.; Groshens, T.J.; Long, J.R.; Harvey, B.G. Hightemperature Magnetic Blocking and Magneto-structural Correlations in a Series of Dysprosium(III) Metallocenium Single-molecule Magnets. Chem. Sci. 2018, 9, 8492-8503.

29. Ungur, L.; Thewissen, M.; Costes, J.P.; Wernsdorfer, W.; Chibotaru, L.F. Interplay of Strongly Anisotropic Metal Ions in Magnetic Blocking of Complexes. Inorg. Chem. 2013, 52, 6328-6337.

30. Liu, Junliang; Chen, Yancong; Tong, Mingliang. Symmetry Strategies for High Performance Lanthanide-Based Single-molecule Magnets. Chem. Soc. Rev. 2018, 47, 2431-2453. 
31. Jin, Pengbo; Zhai, Yuanqi; Yu, Kexin; Winpenny, R.E.P.; Zheng, Yanzhen. Dysprosiacarboranes as Organometallic Single-molecule Magnets. Angew. Chem. Int. Ed. 2020, 59, 9350-9354.

32. Chiesa, A.; Cugini, F.; Hussain, R.; Macaluso, E.; Allodi, G.; Garlatti, E.; Giansiracusa, M.; Goodwin, C.A.P; Ortu, F.; Reta, D.; Skelton, M.J.; Guidi, T.; Santini, P.; Solzi, M.; Renzi, R.D.; Mills, D.P.; Chilton, N.F.; Carretta, S. Understanding Magnetic Relaxation in Single-ion Magnets with High Blocking Temperature. Phys. Rev. B. 2020, 101, 174402-9.

33. Chen, Yancong; Liu, Junliang; Ungur, L.; Liu, Jiang; Li, Quanwen; Wang, Longfei; Ni, Zhaoping; Chibotaru, L. F.; Chen, Xiaoming; Tong, Mingliang. Symmetry-supported Magnetic Blocking at $20 \mathrm{~K}$ in Pentagonal Bipyramidal Dy(III) Single-ion Magnets. J. Am. Chem. Soc. 2016, 138, 2829-2837.

34. Jiang, Shangda; Wang, Bingwu; Sun, Haoling; Wang, Zheming; Gao, Song. An Organometallic Singleion Magnet. J. Am. Chem. Soc. 2011, 133, 4730-4733.

35. Gould, C.A.; Mu, E.; Vieru, V.; Darago, L.E.; Chakarawet, K.; Gonzalez, M.I.; Demir, S.; Long, J.R.; Substituent Effects on Exchange Coupling and Magnetic Relaxation in 2,2'-Bipyrimidine Radical-Bridged Dilanthanide Complexes. J. Am. Chem. Soc. 2020, 142, 21197-21209.

36. Gregson, M.; Chilton, N.F.; Ariciu, A.M.; Tuna, F.; Crowe, I.; Lewis, W.; Blake, A.J.; Collison, D.; McInnes, E.J.L.; Winpenny, R.E.P.; Liddle, S.; A Monometallic Lanthanide Bis (methanediide) Single Molecule Magnet with a Large Energy Barrier and Complex Spin Relaxation Behaviour. Chem. Sci. 2016, 7, 155165.

37. Canaj, A.B.; Singh, M.K.; Wilson, C.; Rajaraman, G.; Murrie, M. Chemical and in Silico Tuning of the Magnetisation Reversal Barrier in Pentagonal Bipyramidal Dy (iii) Single-ion Magnets. Chem. Commun. 2018, 54, 8273-8276. 
38. Liu, Yang; Chen, Yancong; Liu, Jiang; Chen, Wenbin; Huang, Guozhang; Wu, Siguo; Wang, Jing; Liu, Junliang; Tong, Mingliang. Cyanometallate-bridged Didysprosium Single-molecule Magnets Constructed with Single-ion Magnet Building Block. Inorg. Chem. 2020, 59, 687-694.

39. Meng, Yinshan; Xiong, Jin; Yang, Muwen; Qiao, Yusen; Zhong, Zhiqiang; Sun, Haoling; Han, Junbo; Liu, Tao; Wang, Bingwu; Gao, Song. Experimental Determination of Magnetic Anisotropy in Exchange-bias Dysprosium Metallocene Single-Molecule Magnets. Angew. Chem. Int. Ed. 2020, 59, 13037-13043.

40. Demir, S.; Gonzalez, M.I.; Darago, L.E.; Evans, W.J.; Long, J.R. Giant Coercivity and High Magnetic Blocking Temperatures for $\mathrm{N}_{2}{ }^{3-}$ Radical-bridged Dilanthanide Complexes upon Ligand Dissociation. Nat. Commun. 2017, 8, 2144-2147.

41. Rinehart, J.D.; Fang, M.; Evans, W.J.; Long, J.R. A N $2^{3-}$ Radical-bridged Terbium Complex Exhibiting Magnetic Hysteresis at 14 K. J. Am. Chem. Soc. 2011, 133, 14236-14239.

42. Liu, Fupin; Velkos, G.; Krylov, D.S.; Spree, L.; Zalibera, M.; Ray, R.; Samoylova, N.A.; Chen, C.H.; Rosenkranz, M.; Schiemenz, S.; Ziegs, F.; Nenkov, K.; Kostanyan, A.; Greber, T.; Wolter, A.U.B.; Richter, M.; Büchner, B.; Avdoshenko, S.M.; Popov, A.A. Air-Stable Redox-active Nanomagnets with Lanthanide Spins Radical-bridged by a Metal-metal Bond. Nat. Commun. 2019, 10: 571, 10.1038/s41467-019-08513-6.

43. Liu, Fupin; Krylov, D.S.; Spree, L.; Avdoshenko, S.M.; Samoylova, N.A.; Rosenkranz, M.; Kostanyan, A.; Greber, T.; Wolter, A.U.B.; Büchner, B.; Popov, A.A. Single Molecule Magnet with an Unpaired Electron Trapped Between two Lanthanide Ions Inside a Fullerene. Nat. Commun. 2017, 8: 16098, $10.1038 /$ ncomms16098.

44. Zhang, Peng; Perfetti, M.; Kern, M.; Hallmen, P.P.; Ungur, L.; Lenz, S.; Ringenberg, M.R.; Frey, W.; Stoll, H.; Rauhut, G.; Slageren, J.V. Exchange Coupling and Single Molecule Magnetism in Redox-rctive Tetraoxolene-bridged Dilanthanide Complexes. Chem. Sci. 2018, 9, 1221-1230. 
45. Liu, Xiangwen; Wu, Zhuo; Chen, Jitun; Li, Li; Chen, Peng; Sun, Wenbin. Regulating the Single-molecule Magnetic Properties of Phenol Oxygen-bridged Binuclear Lanthanide Complexes Through the Electronic and Spatial Effect of the Substituents. Inorg. Chem. Front. 2020, 7, 1229-1238.

46. Xiong, Jin; Ding, Haiyan; Meng, Yinshan; Gao, Song. Hydroxide-bridged Five-coordinate DylII Singlemolecule Magnet Exhibiting the Record Thermal Relaxation Barrier of Magnetization Among Lanthanide-only Dimers. Chem. Sci. 2016, 8, 1288-1294.

47. Han, Tian; Ding, Yousong; Zhai, Yuanqi; Yu, Kexin; Chilton, N.F.; Zheng, Yanzhen. A Dichlorido-bridged Dinuclear Dy(III) Single Molecule Magnet with an Effective Energy Barrier Larger than 600 K. Chem. Commun. 2019, 55, 7930-7933.

48. Wang, Jin; Li, Quanwen; Wu, Siguo; Chen, Yancong; Wan, Ruichen; Huang, Guozhang; Liu, Yang; Liu, Junliang; Reta, D.; Marcus J. G.; Wang, Zhenxing; Chilton, N. F.; Tong, Mingliang; Opening Magnetic Hysteresis by Axial Ferromagnetic Coupling: From Mono-Decker to Double-Decker Metallacrown. Angew. Chem. Int. Ed. 2021, 60, 5299-5306.

49. Spree, L.; Schlesier, C.; Kostanyan, A.; Westerstrçm, R.; Greber, T.; Bechner, B.; Avdoshenko, S.M.; Popov, A.A. Single-molecule Magnets $\mathrm{DyM}_{2} \mathrm{~N} @ \mathrm{C}_{80}$ and $\mathrm{Dy}_{2} \mathrm{MN} @ \mathrm{C}_{80}(\mathrm{M}=\mathrm{Sc}$, Lu): The Impact of Diamagnetic Metals on Dy3+ Magnetic Anisotropy, Dy-Dy Coupling, and Mixing of Molecular and Lattice Vibrations. Chem. Eur. J. 2020, 26, 2436-2449.

50. Hilgar, J.; Flores, B.S.; Rinehart, J.D. Ferromagnetic Coupling in a Chloride-bridged Erbium Singlemolecule Magnet. Chem. Commun. 2017, 53, 7322-7324. 
Table of Contents Graphic

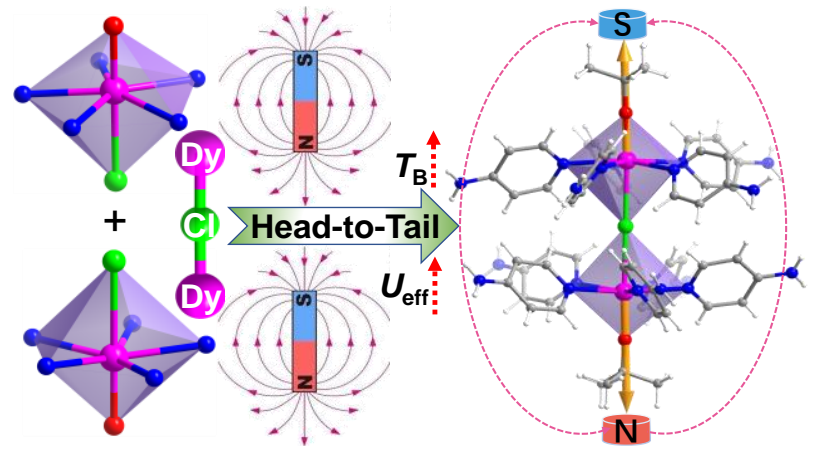

